\title{
Interaction of Parametric and Forced Vibrations in High Speed Rotor-Bearing-Systems
}

\author{
BERNHARDT WEYH \\ Dr.-Ing., University of Duisburg, Dept. Mechanical Engineering/Mechanics, Lotharstr.1, 47048 Duisburg, GERMANY
}

(Received 5 August 1994)

\begin{abstract}
In this paper the interaction of periodic parameter excitation with forced excitations of in general non commensurable basic frequencies is under consideration. The method of enlarged systems serves as a toolbox, where the central idea of the method and the structure of the enlarged system are in the same way derived as the complete homogeneous and the manifold of inhomogeneous solutions are discussed. The application to a realisation of a high speed twin-disc rotor-bearing-system of a textile spinning turbine illustrates the resonance effects that haven't been characterized before.
\end{abstract}

Keywords: Rotor Vibration, Twin-Disc Bearing, Spinning Turbine, Bearing-Inhomogeneity, Parameter Excitation, Resonance Phenomena

\section{MOTIVATION AND INTRODUCTION}

The development of machines emphasizes single functional units or modules. Priority is given to projects which increase machine performance and productivity. These developments do not always comply with the rules of traditional mechanical engineering. New effects and phenomena that haven't been analysed before must be considered. However this can be supported and accelerated by modern sophisticated modelling techniques and computer simulations.

A part of the development of the heart of a rotor spinning machine - the high speed rotor bearing of the spinbox-turbine, see Hanzel [1985], installed in the latest machine generation, see Socha et al. [1991], - will be considered here. Vibration phenom- ena occurring at very high speeds (80000-130000 rpm) called for modification of the twin-disc bearing. Weck et al. [1984] have supposed that the elastic inhomogeneities of the twin-disc coating and the driving belt have effects on rotor vibration. A simple plain model will lead to a set of inhomogeneous differential equations with periodic parameter excitation and periodic forcing, first mentioned by Weyh [1993], where the basic frequencies are quite different and normally non commensurable.

Vibrations of linear parameter excited systems are mainly analysed by simulation techniques (initial value problems). A detailed survey of these methods for the stability problem including literature specifications are given in Friedemann et al. [1977] and Weyh et al. [1991]. The quality of the numerical results is essentially dependant on the eigen-values, the 
parameter excitation frequency and, if present, the forced excitation frequencies. If the eigen-values are situated far from each other, the integration-time increases in the same way as the integration interval increases if parameter and/or excitation frequencies are different. In general long-time simulations are necessary to analyse the steady state vibrations, because it must be guaranteed that the eigen-vibrations have been faded away. A wide numerical study has to be done normally, to find out general information about solution manifold, phenomena and parameterdependance, see for example Diekhans [1981] and Laschet [1988].

These time-consuming problems can be avoided when replacing the time-variant basic system by a time-invariant description of higher order with the same or in the case of approximation nearly the same quality of solution. This idea can be realized by a simple substitution of the system states.

The method is quite similar to Hill and Bolotin (Bolotin [1964]) for the homogeneous part, because the enlarged system matrices are equivalent to the generalized Hill matrices introduced in Weyh [1989, 1990], where the approximation error and the convergence problems are widely discussed. Here the new idea serves for interpretation and generalization of the method. It enables us to find general resonance conditions of the interaction of parameter and forced excited vibrations with commensurable or non commensurable basic frequencies.

\section{TRANSFORMATION AND \\ SYSTEM-ENLARGEMENT}

\section{Basic System}

With the help of the $f \times 1$-dimensional generalized coordinate-vector

$$
\mathbf{y}=\left(y_{1}, y_{2}, \ldots, y_{f}\right)^{T}
$$

a general structure of mathematical models of periodic parameter excited, inhomogeneous linear systems can be described by

$$
\mathbf{M}(t) \ddot{\mathbf{y}}+\mathbf{P}(t) \dot{\mathbf{y}}+\mathbf{Q}(t) \mathbf{y}=\mathbf{h}(t)
$$

where dots denote derivatives with respect to time $t$. The $T_{p}=2 \pi / \Omega$-periodic, in general real, $f \times f$-system matrices will be represented by finite complex Fourier-matrix-series

$$
\begin{gathered}
\mathbf{M}(t)=\sum_{k=-K}^{K} \mathbf{M}_{k} e^{j k \Omega t}, \jmath \mathbf{P}(t)=\sum_{k=-K}^{K} \mathbf{P}_{k} e^{j k \Omega t}, \\
\mathbf{Q}(t)=\sum_{k=-K}^{K} \mathbf{Q}_{k} e^{\Omega t}
\end{gathered}
$$

with $\jmath=\sqrt{-1}$, to simplify the following procedures. $\mathbf{h}(t)$ stands for the $T_{f}=2 \pi / \eta$-periodic $f \times 1$-forcingvector.

\section{Enlarged System}

The complex substitutions

$$
\mathbf{y}_{p}(t)=\mathbf{y}(t) e^{j p \Omega t}, j p=-P(1) P
$$

$(p=-P(1) P$ stands for all values $p$ of the integer interval $[-P, P])$ transform the basic system (2) under consideration of the complex series of system matrices (3) into $2 P+1$ coupled matrix differential equations of basic order $2 f$, see Weyh [1993] and ViehHweger [1993]. With the help of the hyper-coordinate-vector

$$
\overline{\mathbf{y}}=\left(\mathbf{y}_{-P}^{T}, \ldots, \mathbf{y}_{-1}^{T}, \mathbf{y}_{0}^{T}, \mathbf{y}_{1}^{T}, \ldots, \mathbf{y}_{P}^{T}\right)^{T}
$$

the derived enlarged system can be represented in a compact linear hyper-matrix form

$$
\overline{\mathbf{M}} \overline{\mathbf{y}}+\overline{\mathbf{P}} \overline{\mathbf{y}}+\overline{\mathbf{Q}} \overline{\mathbf{y}}+\overline{\mathbf{g}}_{P}(t, \overline{\mathbf{y}}, \overline{\mathbf{y}}, \overline{\mathbf{y}})=\overline{\mathbf{h}}(t)
$$

of the order $N=2 f(2 P+1)$ and is exactly equivalent to the basic system (2). The periodic parameter excited, ordinary differential system (6) consists on the left side of a constant and a time-variant part. The structure of the constant part can simply be seen from the $N \times N$-dimensional coefficient-matrices in the sub-matrix representations given for an example in 
Table I. The hyper-matrices $\overline{\mathbf{M}}, \overline{\mathbf{P}}, \overline{\mathbf{Q}}$ can be constructed algorithmically by means of the system coefficient matrices $\mathbf{M}_{k}, \mathbf{P}_{k}, \mathbf{Q}_{k}$ of the basic system (2) given in (3). The hyper-matrices are closely connected with the Hill matrix approximations by Bolotin [1964] and are exactly the same as given in the generalized Hill matrix approximation by Weyh $[1989,1990]$ if using real instead of complex Fourier-series representations in (3) and (4). The timevarying part of (6) consists of $T_{p}$-periodic coefficientmatrices of higher harmonic orders-and this is the speciality of the enlarged system-only higher than the substitution order $P$. The $f \times 1$-dimensional subvectors

$$
\mathbf{h}_{p}(t)=\mathbf{h}(t) e^{j \Omega t}, J p=-P(1) P
$$

of the hyper-vector of inhomogeneity on the right side of (6)

$\overline{\mathbf{h}}(t)=\left(\mathbf{h}_{-P}^{T}(t), \ldots, \mathbf{h}_{-1}^{T}(t), \mathbf{h}_{0}^{T}(t), \mathbf{h}_{1}^{T}(t), \ldots, \mathbf{h}_{P}^{T}(t)\right)^{T}$ can be interpreted as amplitude modulations of the forcing function of the basic system (2) on the harmonics of the parameter excitation as carrier frequency.

\section{Approximate System}

By neglecting the higher harmonic parameter excitation $\overline{\mathbf{g}}_{P}(\bullet)$, equation (6) reduces to a time-invariant matrix equation of the order $N$

$$
\overline{\mathbf{M}} \overline{\mathbf{y}}+\overline{\mathbf{P}} \overline{\mathbf{y}}+\overline{\mathbf{Q}} \overline{\mathbf{y}}=\overline{\mathbf{h}}(t)
$$

A detailed discussion of the approximation errors and the convergence of homogeneous solutions can be found in the same way in Weyh [1989] as a comparison with Floquet-solutions in Müller et al. [1976] and Yakubovich et al. [1975].

TABLE I Example of the Structure of the Coefficient-Matrices $\overline{\mathbf{M}}, \overline{\mathbf{P}}, \overline{\mathbf{Q}}$ of the Enlarged System with $K=3, P=2$.

$$
\left[\begin{array}{ccccc}
\mathbf{M}_{0} & \mathbf{M}_{1} & \mathbf{M}_{2} & \mathbf{M}_{3} & \mathbf{0} \\
\mathbf{M}_{-1} & \mathbf{M}_{0} & \mathbf{M}_{1} & \mathbf{M}_{2} & \mathbf{M}_{3} \\
\mathbf{M}_{-2} & \mathbf{M}_{-1} & \mathbf{M}_{0} & \mathbf{M}_{1} & \mathbf{M}_{2} \\
\mathbf{M}_{-3} & \mathbf{M}_{-2} & \mathbf{M}_{-1} & \mathbf{M}_{0} & \mathbf{M}_{1} \\
\mathbf{0} & \mathbf{M}_{-3} & \mathbf{M}_{-2} & \mathbf{M}_{-1} & \mathbf{M}_{0}
\end{array}\right]
$$

$$
\left[\begin{array}{ccccc}
\mathbf{P}_{0}+j 4 \Omega \mathbf{M}_{0} & \mathbf{P}_{1}+\jmath 2 \Omega \mathbf{M}_{1} & \mathbf{P}_{2} & \mathbf{P}_{3}-j 2 \Omega \mathbf{M}_{3} & \mathbf{0} \\
\mathbf{P}_{-1}+j 4 \Omega \mathbf{M}_{-1} & \mathbf{P}_{0}+j 2 \Omega \mathbf{M}_{0} & \mathbf{P}_{1} & \mathbf{P}_{2}-j 2 \Omega \mathbf{M}_{2} & \mathbf{P}_{3}-j 4 \Omega \mathbf{M}_{3} \\
\mathbf{P}_{-2}+j 4 \Omega \mathbf{M}_{-2} & \mathbf{P}_{-1}+j 2 \Omega \mathbf{M}_{-1} & \mathbf{P}_{0} & \mathbf{P}_{1}-j 2 \Omega \mathbf{M}_{1} & \mathbf{P}_{2}-j 4 \Omega \mathbf{M}_{2} \\
\mathbf{P}_{-3}+j 4 \Omega \mathbf{M}_{-3} & \mathbf{P}_{-2}+\jmath 2 \Omega \mathbf{M}_{-2} \mathbf{P}_{-1} & \mathbf{P}_{0}-j 2 \Omega \mathbf{M}_{0} & \mathbf{P}_{1}-j 4 \Omega \mathbf{M}_{1} \\
\mathbf{0} & \mathbf{P}_{-3}+j 2 \Omega \mathbf{M}_{-3} & \mathbf{P}_{-2} & \mathbf{P}_{-1}-j 2 \Omega \mathbf{M}_{-1} \mathbf{P}_{0}-j 4 \Omega \mathbf{M}_{0}
\end{array}\right]
$$

$$
\left[\begin{array}{ccccc}
\mathbf{Q}_{0}+\jmath 2 \Omega \mathbf{P}_{0}-4 \Omega^{2} \mathbf{M}_{0} & \mathbf{Q}_{1}+\jmath \Omega \mathbf{P}_{1}-\Omega^{2} \mathbf{M}_{1} & \mathbf{Q}_{2} & \mathbf{Q}_{3}-\jmath \Omega \mathbf{P}_{3}-\Omega^{2} \mathbf{M}_{3} & \mathbf{0} \\
\mathbf{Q}_{-1}+\jmath 2 \Omega \mathbf{P}_{-1}-4 \Omega^{2} \mathbf{M}_{-1} & \mathbf{Q}_{0}+\jmath \Omega \mathbf{P}_{0}-\Omega^{2} \mathbf{M}_{0} & \mathbf{Q}_{1} & \mathbf{Q}_{2}-\jmath \Omega \mathbf{P}_{2}-\Omega^{2} \mathbf{M}_{2} & \mathbf{Q}_{3}-\jmath 2 \Omega \mathbf{P}_{3}-4 \Omega^{2} \mathbf{M}_{3} \\
\mathbf{Q}_{-2}+\jmath 2 \Omega \mathbf{P}_{-2}-4 \Omega^{2} \mathbf{M}_{-2} & \mathbf{Q}_{-1}+\jmath \Omega \mathbf{P}_{-1}-\Omega^{2} \mathbf{M}_{-1} & \mathbf{Q}_{0} & \mathbf{Q}_{1}-\jmath \Omega \mathbf{P}_{1}-\Omega^{2} \mathbf{M}_{1} & \mathbf{Q}_{2}-\jmath 2 \Omega \mathbf{P}_{2}-4 \Omega^{2} \mathbf{M}_{2} \\
\mathbf{Q}_{-3}+\jmath 2 \Omega \mathbf{P}_{-3}-4 \Omega^{2} \mathbf{M}_{-3} & \mathbf{Q}_{-2}+\jmath \Omega \mathbf{P}_{-2}-\Omega^{2} \mathbf{M}_{-2} \mathbf{Q}_{-1} & \mathbf{Q}_{0}-\jmath \Omega \mathbf{P}_{0}-\Omega^{2} \mathbf{M}_{0} & \mathbf{Q}_{1}-\jmath 2 \Omega \mathbf{P}_{1}-4 \Omega^{2} \mathbf{M}_{1} \\
\mathbf{0} & \mathbf{Q}_{-3}+\jmath \Omega \mathbf{P}_{-3}-\Omega^{2} \mathbf{M}_{-3} & \mathbf{Q}_{-2} \mathbf{Q}_{-1}-\jmath \Omega \mathbf{P}_{-1}-\Omega^{2} \mathbf{M}_{-1} \mathbf{Q}_{0}-\jmath 2 \Omega \mathbf{P}_{0}-4 \Omega^{2} \mathbf{M}_{0}
\end{array}\right]
$$




\section{THEORETICAL CONCLUSIONS}

Instead of considering parameter excited differential equations (6) of order $2 f$, constant systems (9) of order $N$ can be solved. The complete solution of the approximate system can be calculated-and this is the essential advantage - by the well known algorithms of constant systems. In particular the following conclusions can be derived:

\section{Stability Problem}

The eigen-value problem of the approximate system

$$
\left(\overline{\mathbf{M} \lambda^{2}}+\overline{\mathbf{P} \lambda}+\overline{\mathbf{Q}}\right) \overline{\mathbf{a}}=\overline{\mathbf{0}}
$$

leads to $l=1(1) N$ eigen-values

$$
\bar{\lambda}_{l} \equiv \bar{\delta}_{i, j}+j \omega_{i, j}, j i=-f(1) f \neq 0, j j=-P(1) P
$$

It can be shown (for a detailed derivation see Naab et al. [1988], Neumann et al. [1994]) that the eigenvalues coincide with the $2 f$ characteristic exponents $\delta_{i}$ $+\jmath \omega_{i}$ of the basic system (2) except for multiple of the parameter excitation frequency $\Omega$ and approximation errors $\bar{\Delta}_{i, j}$ in the form

$$
\delta_{i}+\jmath\left(\omega_{i}+j \Omega\right)=\bar{\delta}_{i, j}+\bar{j}_{i, j}+\bar{\Delta}_{i, j}
$$

From the afore-mentioned connection it follows, that the stability conditions of the periodic system (2), normally analysed with the help of the characteristic multiplier method (Weyh et al. [1991]), are directly recognizable by the real parts of the eigen-values of (10) (see Müller et al. [1976]) here in the chosen approximation. The system (2) is:

- asymptotically stable, if all eigen-values of (10) have negative real parts

$$
\operatorname{Re} \bar{\lambda}_{l}<0, J l=1(1) N
$$

- unstable, if at least one eigen-value of (10) has a positive real part

$$
\operatorname{Re} \bar{\lambda}_{l}>0, \text { for one } l
$$

Only for critical eigen-values $\bar{\lambda}_{m}$ of (10) with $\operatorname{Re} \bar{\lambda}_{m}$ $=0$, two different cases must be distinguished.

Let $v_{m}$ stand for the multiplicity of the eigen-value with a vanishing real part and $d_{m}=N-\operatorname{rank}\left(\overline{\mathbf{M}}^{2}+\right.$ $\overline{\mathbf{P} \lambda}+\overline{\mathbf{Q}})$ stand for the defect of the accompanying characteristic matrix. If $\operatorname{Re} \bar{\lambda}_{l} \leq 0$ with $l=1(1) N$ and $\operatorname{Re} \bar{\lambda}_{m}=0$, then the system (2) is:

- limit stable for $d_{m}=v_{m}$ otherwise

- unstable for $d_{m}<v_{m}$.

\section{Limit Stability Conditions and Parametric Resonances}

It follows from (12), that all real parts $\bar{\delta}_{i, j}$ of the eigen-values of the approximate system (9) exist in a $2 P+1$ times multiplicity, as in the critical case. If $\omega_{i, j}, \omega_{i, k}$ are two imaginary parts of one critical eigenvalue multiplicity of the approximate system, then the connection with the imaginary parts of the eigenvalues of the basic system

$$
\begin{gathered}
\bar{\omega}_{i, j} \approx \omega_{i}+j \Omega, \bar{\omega}_{i, k} \approx \omega_{i}+k \Omega, \\
i \in-f(1) f \neq 0, \jmath j, k \in-\mathrm{P}(1) \mathrm{P}
\end{gathered}
$$

holds if the approximation errors $\bar{\Delta}_{i, j}, \bar{\Delta}_{i, k}$ are small. From the real structure of the technical starting problem it follows, that a complex eigen-value is followed by its conjugate $\omega_{i}=-\omega_{-i}$. A critical pair of eigenvalues of one critical eigen-value multiplicity of the approximate system can then become multiple in the form

$$
\bar{\omega}_{i, j}=\bar{\omega}_{-i, k}
$$


and this leads to the well known condition, see Yakubovich et al. [1975], of main-parametric or parametric resonance

$$
\omega_{i}=\frac{m}{2} \Omega, \jmath m=k-j
$$

Taking into account $\bar{\omega}_{l, k}$ as an imaginary part of a second critical eigen-value multiplicity, the equality

$$
\bar{\omega}_{i, j}=\bar{\omega}_{-l, k}
$$

leads to the combination resonance condition of the sum type

$$
\omega_{i}+\omega_{l}=m \Omega, \jmath m=k-j
$$

while using the conjugate of $\omega_{l}$, the last condition stands for the difference type.

\section{Homogeneous Solutions}

The substitution (4) shows, that the required homogeneous solution of the basic system (2) results in

$$
\mathbf{y}_{\text {hom }}(t)=\mathbf{y}_{0}(t)=\sum_{l=1}^{N} \overline{c_{l}} \overline{\mathbf{a}}_{0}^{(l)} e^{-\overline{\lambda_{l} t}}
$$

where the scalars $\overline{c_{l}}$ stand for the integration constants considering the initial conditions while the vectors $\overline{\mathbf{a}}_{0}^{(l)}$ stand for the centre parts of the eigen-vectors of the enlarged system

$$
\left.\overline{\mathbf{a}}^{(l)}=\overline{\mathbf{a}}_{-P}^{(l) T}, \ldots, \overline{\mathbf{a}}_{-1}^{(l) T}, \overline{\mathbf{a}}_{0}^{(l) T}, \overline{\mathbf{a}}_{1}^{(l) T}, \ldots, \overline{\mathbf{a}}_{P}^{(l) T}\right)^{T}, l=1(1) N
$$

\section{Inhomogeneous Solutions}

Inhomogeneous solutions as a result of standard forcing (constant, harmonic, periodic, quasiperiodic,...) of the basic system can be calculated easily. The harmonic excitation including a constant part

$$
\mathbf{h}(t)=\sum_{q=-1}^{1} \mathbf{h}_{q} e^{j \eta t}
$$

serves as an example for the construction of an inhomogeneous solution and leads to an excitation vector of the approximated system

$$
\overline{\mathbf{h}}(t)=\sum_{q=-1}^{1} \sum_{p=-P}^{P} \overline{\mathbf{h}}_{q p} e^{\jmath(q \eta+p \Omega) t}
$$

with constant hyper-amplitude-vectors

$$
\begin{gathered}
\overline{\mathbf{h}}_{q,-1}=\left(\ldots \mathbf{0}^{T}, \mathbf{h}_{q}^{T}, \mathbf{0}^{T}, \mathbf{0}^{T}, \mathbf{0}^{T}, \ldots\right)^{T} \\
\overline{\mathbf{h}}_{q, 0}=\left(\ldots \mathbf{0}^{T}, \mathbf{0}^{T}, \mathbf{h}_{q}^{T}, \mathbf{0}^{T}, \mathbf{0}^{T}, \ldots\right)^{T} \\
\overline{\mathbf{h}}_{q, 1}=\left(\ldots \mathbf{0}^{T}, \mathbf{0}^{T}, \mathbf{0}^{T}, \mathbf{h}_{q}^{T}, \mathbf{0}^{T}, \ldots\right)^{T}
\end{gathered}
$$

In case of non commensurable basic frequencies $\eta, \Omega$ (18) is quasiperiodic, otherwise $2 \pi v / \Omega$-periodic ( $v$ integer). The inhomogeneous solution of the approximate system

$$
\overline{\mathbf{y}}_{\text {inh }}(t)=\sum_{q=-1}^{1} \sum_{p=-P}^{P} \overline{\mathbf{b}}_{q p} e^{\jmath(q \eta+p \Omega) t}
$$

is given by sums of products of amplitude-vectors with the harmonic excitation functions. The unknown amplitude-vectors in (20) result from

$$
\overline{\mathbf{b}}_{q p}=\overline{\mathbf{G}}_{q p}(q \eta+p \Omega) \overline{\mathbf{h}}_{q p}
$$

by using the frequency response matrices

$$
\begin{gathered}
\overline{\mathbf{G}}_{q p}(q \eta+p \Omega) \\
=\left[-(q \eta+p \Omega)^{2} \overline{\mathbf{M}}+\jmath(q \eta+p \Omega)\right. \\
\overline{\mathbf{P}}+\overline{\mathbf{Q}}]^{-1}
\end{gathered}
$$


The corresponding solution of the basic system is then given by the $f \times 1$-dimensional center part of (20).

\section{Resonance Conditions and Interactions}

The special representation of the frequency response matrix

$$
\begin{gathered}
\overline{\mathbf{G}}_{q p}(q \eta+p \Omega) \\
=\frac{\operatorname{adj}\left[-(q \eta+p \Omega)^{2} \overline{\mathbf{M}}+\jmath(q \eta+p \Omega) \overline{\mathbf{P}}+\overline{\mathbf{Q}}\right]}{\operatorname{det}\left[-(q \eta+p \Omega)^{2} \overline{\mathbf{M}}+\jmath(q \eta+p \Omega) \overline{\mathbf{P}}+\overline{\mathbf{Q}}\right]}
\end{gathered}
$$

allows the elementary interpretation of the resonance phenomena and the interaction of parametric with forced excitation.

Large amplitudes (resonances) of the inhomogeneous solutions occur if the denominator of the frequency response matrix representation (23) becomes minimal (vanishes). By comparing the denominator with the characteristic equation of (10), including the eigen-value description (11), general resonance conditions

$$
\bar{\omega}_{i, j}=q \eta+p \Omega
$$

can be derived, which result if considering (12)

$$
\begin{gathered}
\omega_{i}+j \Omega \approx q \eta+p \Omega, i=-f(1) f \neq 0, \\
j, p=-P(1) P, j q=-1(1) 1
\end{gathered}
$$

From equation (25) three different resonance types can be classified:

- forced resonance

$$
\omega_{i}=q \eta, \quad j=p, \quad q \neq 0
$$

eigen-frequency equals harmonic forced frequency,
- main parametric resonance

$$
\omega_{i}=(p-j) \Omega, \jmath \neq p, q=0
$$

eigen-frequency equals harmonics of parametric frequency,

- interaction of forced and parametric resonance

$$
\omega_{i}=q \eta+(p-j) \Omega, \jmath \neq p, q \neq 0
$$

eigen-frequency equals linear combinations of harmonics of forced and parametric frequencies.

\section{APPLICATION TO OPEN-END ROTOR-BEARING-SYSTEMS}

\section{Description of System}

The picture in Figure 1 shows the lower part of a high speed Open-End rotor-bearing with a spinning rotor, carrying the Open-End spinning turbine at its front end, Socha et al. [1991]. The bearing-system consists of two rigid coupled twin discs mounted in two bearing shells which are fixed in a bearing house. The drive of the spinning rotor follows on from a transmission belt crossing the rotor, schematically sketched in Figure 2. (Up to 120 spinning rotors are driven by the same belt from a central drive in one spinning machine.) The necessary tangential belt wrap is produced by the elastic supported billy-roller which presses the rotor between the elastic coating of

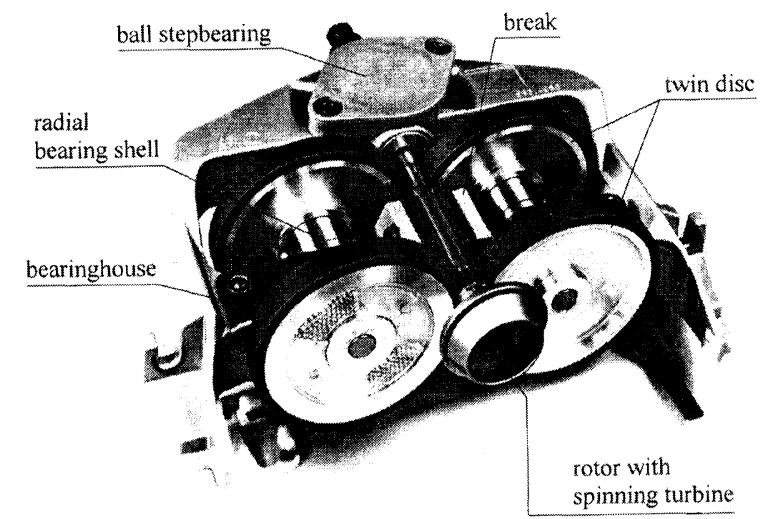

FIGURE 1 Picture of lower part of Open-End spinning box in the latest machine generation. 


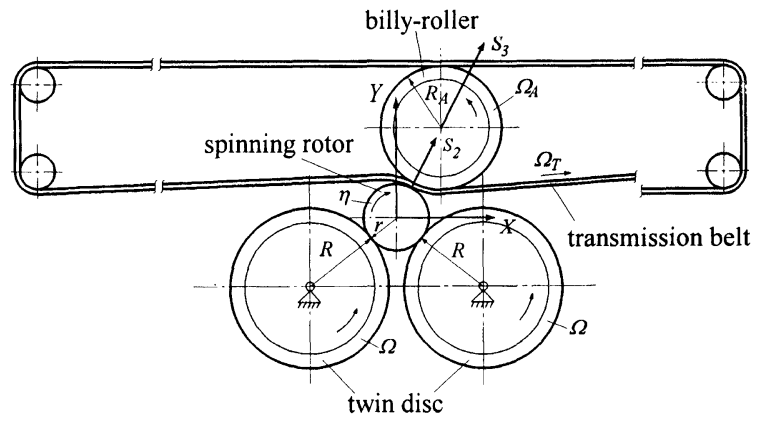

FIGURE 2 Plain sketch of Open-End spinning box with generalised coordinates and characteristic system parameters.

the twin disc pairs for the radial bearing. Due to a minimal deviation from parallelism of the twin disc axis a permanent thrust load acts on the rotor and squeezes its back end against a ball step bearing for axial fixation.

\section{Structure of Model Equation}

For first predictions a simple mechanical, three-mass model with four degrees of freedom, on the base of Figure 2, can be used. The generalized 4-dimensional coordinate-vector, introduced by (1),

$$
\mathbf{y}=\left(X, Y, S_{2}, S_{3}\right)^{T}
$$

describes in this model the horizontal $X$ and the vertical $Y$ displacement of the spinning rotor axis. $S_{2}$ stands for the movement of the acting part of the transmission belt in the direction of contact with the billy-roller, while in the same direction $S_{3}$ represents the motion of the axis of the billy-roller.

The constant parts of the system matrices of (3) are defined by the real symmetric matrices

$$
\begin{gathered}
\mathbf{M}_{0}=\operatorname{diag}\left(m_{1}+m_{u}, m_{1}+m_{u}, m_{2}, m_{3}\right) \\
\mathbf{P}_{0}=\left[\begin{array}{cccc}
p_{11}^{0} & p_{12}^{0}-d_{21} \cos \gamma & 0 \\
& p_{22}^{0} & -d_{21} \sin \gamma & 0 \\
& & d_{21}+d_{32} & -d_{32} \\
\text { sym } & & d_{32}+d_{3}
\end{array}\right]
\end{gathered}
$$

$$
\mathbf{Q}_{0}=\left[\begin{array}{cccc}
q_{11}^{0} & q_{12}^{0}-c_{21} \cos \gamma & 0 \\
& q_{22}^{0}-c_{21} \sin \gamma & 0 \\
& c_{21}+c_{32} & -c_{32} \\
\operatorname{sym} & & c_{32}+c_{3}
\end{array}\right]
$$

where the abbreviations of elements of the damping matrix are given with the expressions

$$
\begin{gathered}
p_{11}^{0}=\left(d_{t 1}^{0}+d_{t 2}^{0}\right) \cos ^{2} \delta+d_{21} \cos ^{2} \gamma \\
p_{12}^{0}=\left(d_{t 1}^{0}-d_{t 2}^{0}\right) \sin \delta \cos \delta+d_{21} \sin \gamma \cos \gamma \\
p_{22}^{0}=\left(d_{t 1}^{0}+d_{t 2}^{0}\right) \sin ^{2} \delta+d_{21} \sin ^{2} \gamma
\end{gathered}
$$

and, by exchanging $d$ for $c$ formally in the expressions of $p_{i j}^{0}$, the elements of the stiffness matrix $q_{i j}^{0}$ are introduced.

The parameter excitation of the system results from the elastic inhomogeneities of the twin disc coatings and can be represented by periodically varying damping and stiffness coefficients

$$
\begin{gathered}
d_{t 1 / t 2}=d_{t 1 / t 2}^{0}\left[1+\sum_{k=1}^{K}\left(\alpha_{t 1 / t 2}^{c k} \cos k \Omega t\right.\right. \\
\left.\left.+\alpha_{t 1 / t 2}^{s k} \sin k \Omega t\right)\right] \\
c_{t 1 / t 2}=c_{t 1 / t 2}^{0}\left[1+\sum_{k=1}^{K}\left(\beta_{t 1 / t 2}^{c k} \cos k \Omega t\right.\right. \\
\left.\left.+\beta_{t 1 / t 2}^{s k} \sin k \Omega t\right)\right]
\end{gathered}
$$

with the basic frequency $\Omega$ of the twin discs and the small number $K=3$ of harmonics as given in the example in Table I. Considering this arrangement, the time-variant parts of the system matrices are given by the complex symmetric coefficient-matrices

$$
\begin{gathered}
\mathbf{M}_{k}=\mathbf{0}, \\
\mathbf{P}_{k}=\left[\begin{array}{llll}
p_{11}^{k} & p_{12}^{k} & 0 & 0 \\
& p_{22}^{k} & 0 & 0 \\
& 0 & 0 \\
\text { sym } & & 0
\end{array}\right], \\
\mathbf{Q}_{k}=\left[\begin{array}{lrrr}
q_{11}^{k} & q_{12}^{k} & 0 & 0 \\
& q_{22}^{k} & 0 & 0 \\
& & 0 & 0 \\
\text { sym } & & 0
\end{array}\right]
\end{gathered}
$$


of the trigonometric matrix-series (3), with the abbreviations of the complex elements

$$
\begin{aligned}
p_{11}^{k} & =\left[\left(d_{t 1}^{0} \alpha_{t 1}^{c k}+d_{t 2}^{0} \alpha_{t 2}^{c k}\right) \mp \jmath\left(d_{t 1}^{0} \alpha_{t 1}^{s k}+d_{t 2}^{0} \alpha_{t 2}^{s k}\right)\right] \cos ^{2} \delta \\
p_{12}^{k} & =\left[\left(d_{t 1}^{0} \alpha_{t 1}^{c k}-d_{t 2}^{0} \alpha_{t 2}^{c k}\right)\right. \\
& \left.\mp \jmath\left(d_{t 1}^{0} \alpha_{t 1}^{s k}-d_{t 2}^{0} \alpha_{t 2}^{s k}\right)\right] \sin \delta \cos \delta \\
p_{22}^{k} & =\left[\left(d_{t 1}^{0} \alpha_{t 1}^{c k}+d_{t 2}^{0} \alpha_{t 2}^{c k}\right) \mp \jmath\left(d_{t 1}^{0} \alpha_{t 1}^{s k}+d_{t 2}^{0} \alpha_{t 2}^{s k}\right)\right] \sin ^{2} \delta
\end{aligned}
$$

and $q_{i, j}^{k}$ by exchanging $\alpha$ for $\beta$ and $d$ for $c$ respectively as mentioned before. The minus or plus sign of (31) stands for positive or negative values of $k \neq 0$.

Outer system excitation results from the centrifugal force of the imbalance

$$
F_{u}=m_{u} e \eta^{2}
$$

dominantly caused by the spinning process. The complex vector of excitation forces (17) consists of a constant $q=0$ and a harmonic $q=-1,1$ part

$$
\begin{aligned}
\mathbf{h}_{-1} & =\left(-\frac{1}{2} F_{u},-\jmath \frac{1}{2} F_{u}, 0,0\right)^{T} \\
\mathbf{h}_{0} & =\left(0,0,-F_{2},-F_{3}\right)^{T} \\
\mathbf{h}_{1} & =\left(-\frac{1}{2} F_{u}, \jmath \frac{1}{2} F_{u}, 0,0\right)^{T}
\end{aligned}
$$

as a result of the initial tensions $F_{2}, F_{3}$ and the uniform rotation $\varphi=\eta t$ of the spinning turbine. It is assumed that the system drive realizes motions without slip and that the spinning turbine rotates at a constant angular velocity $\dot{\varphi}=\eta$. Therefore the rotational frequency of the twin disc

$$
\Omega=\eta r / R
$$

\begin{tabular}{|c|c|c|c|}
\hline mass & $m_{1}$ & rotor/turbine & $0.112 \mathrm{~kg}$ \\
\hline & $m_{u}$ & imbalance & $1.010^{-5} \mathrm{~kg}$ \\
\hline & $m_{2}$ & active part of transmission belt & $0.03 \mathrm{~kg}$ \\
\hline & $m_{3}$ & billy-roller & $0.3 \mathrm{~kg}$ \\
\hline \multirow[t]{7}{*}{ damping } & $d_{t 1 / t 2}^{0}$ & twin disc coating (mean value) & $15.0 / 15.0 \mathrm{~kg} / \mathrm{s}$ \\
\hline & $\alpha_{t 1}^{c k}$ & twin disc coating (varying cos-coeff.) & $+0.3 /+0.1 /+0.05$ \\
\hline & $\alpha_{t 2}^{c k}$ & twin disc coating (varying cos-coeff.) & $-0.3 /-0.1 /-0.05$ \\
\hline & $\alpha_{t 1}^{s k}$ & twin disc coating (varying sin-coeff.) & $0.0 / 0.0 / 0.0$ \\
\hline & $\alpha_{t 2}^{s k}$ & twin disc coating (varying sin-coeff.) & $0.0 / 0.0 / 0.0$ \\
\hline & $d_{21 / 32}$ & belt coating (normal) & $15.0 / 15.0 \mathrm{~kg} / \mathrm{s}$ \\
\hline & $d_{3}$ & billy-roller damper & $7.45 \mathrm{~kg} / \mathrm{s}$ \\
\hline \multirow[t]{7}{*}{ stiffness } & $c_{t 1 / t 2}^{0}$ & twin disc coating (mean value) & $2.010^{6} / 2.010^{6} \mathrm{~kg} / \mathrm{s}^{2}$ \\
\hline & $\beta_{t 1}^{c k}$ & twin disc coating (varying cos-coeff.) & $+0.3 /+0.1 /+0.05$ \\
\hline & $\beta_{t 2}^{c k}$ & twin disc coating (varying cos-coeff.) & $-0.3 /-0.1 /-0.05$ \\
\hline & $\beta_{t 2}^{s k}$ & twin disc coating (varying sin-coeff.) & $0.0 / 0.0 / 0.0$ \\
\hline & $\beta_{t 1}^{s k}$ & twin disc coating (varying sin-coeff.) & $0.0 / 0.0 / 0.0$ \\
\hline & $c_{21 / 32}$ & belt coating (normal direction) & $1.010^{6} / 1.010^{6} \mathrm{~kg} / \mathrm{s}^{2}$ \\
\hline & $c_{3}$ & billy-roller spring & $1.510^{5} \mathrm{~kg} / \mathrm{s}^{2}$ \\
\hline force & $F_{2 / 3}$ & initial tension & $15 / 15 N$ \\
\hline \multirow[t]{4}{*}{ geometry } & $\delta / \gamma$ & position angles & $0.48 / 0.628 \mathrm{rad}$ \\
\hline & $e$ & excentricity of imbalance & $0.017 \mathrm{~m}$ \\
\hline & $r$ & radius of spinning rotor & $0.0045 m$ \\
\hline & $R$ & radius of twin disc & $0.035 \mathrm{~m}$ \\
\hline
\end{tabular}

is directly connected with the rotor frequency. The relation $r / R$ will later decide on periodic (commensurable frequencies) or quasiperiodic (non commensurable frequencies) inhomogeneous solutions.

The system parameters, see Table II, point to es-

TABLE II Description and characteristic values of system parameters of rotor-bearing-model 


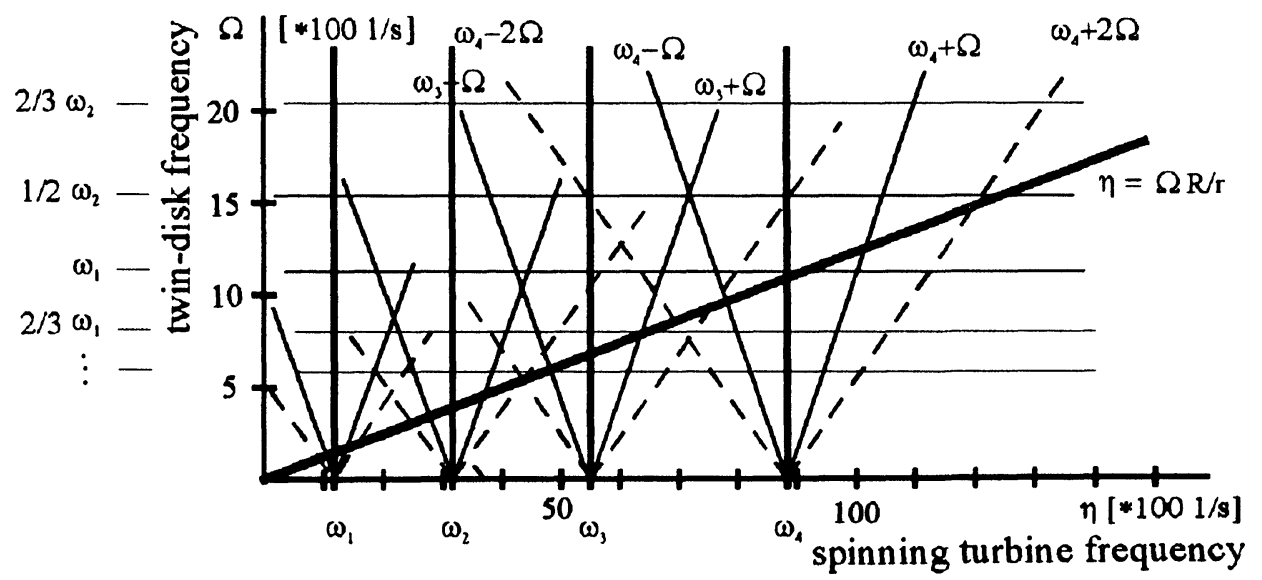

FIGURE 3 Visualization of resonance conditions in the parameter space: twin-disc frequency $\Omega$ against spinning turbine frequency $\eta$.

sential background information about the modelling process.

\section{Results}

Resonance points For quick predictions and a first orientation in the parameter space of interest-parametric frequency via forced frequency-the resonance conditions (13), (14), (25) are sketched in Figure 3 for the spinning system presented before in a simple approximation $j=-1(1) 1, p=-1(1) 1$. Parametric resonance lines are given by the horizontal lines, forced resonance lines by the vertical lines, main parametric resonance lines by some of the horizontal lines, while the skew lines stand for the interaction of forced with parametric resonances. The in-

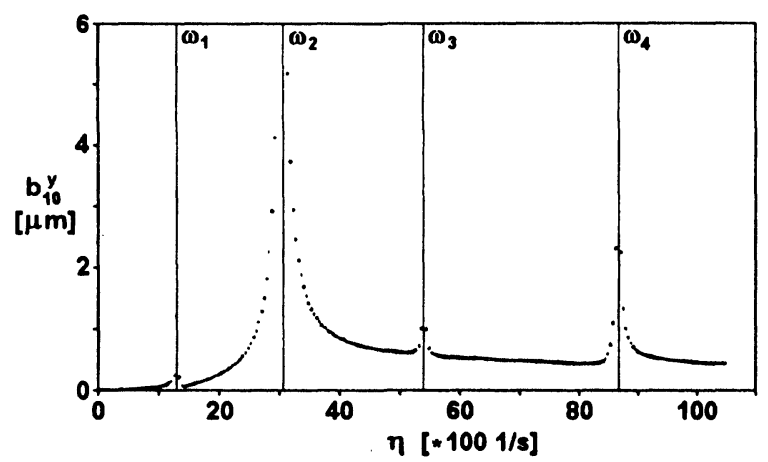

tersections of the described resonance lines with the thick frequency line $\eta=\Omega R / r$, see (34), stand for the points where resonances or large amplitudes can occur. In case of higher approximations further resonances radiate out from the eigen-frequency-points marked on the $\eta$-axis. Normally the importance of the resonances decreases with increasing resonance order $m$ and approximation order $P$. This effect depend on the convergence of harmonic amplitudes of the parameter excitation (28), (29).

Response spectra The solutions of equation (21) with (22) are calculated with a constant step rate $\Delta \eta$ and exemplary the vertical vibrations of the spinning rotor ( $Y$-direction) are plotted point-by-point against the excitation frequency $\eta$ in the following figures. The amplitude and phase curve (of inhomogeneous

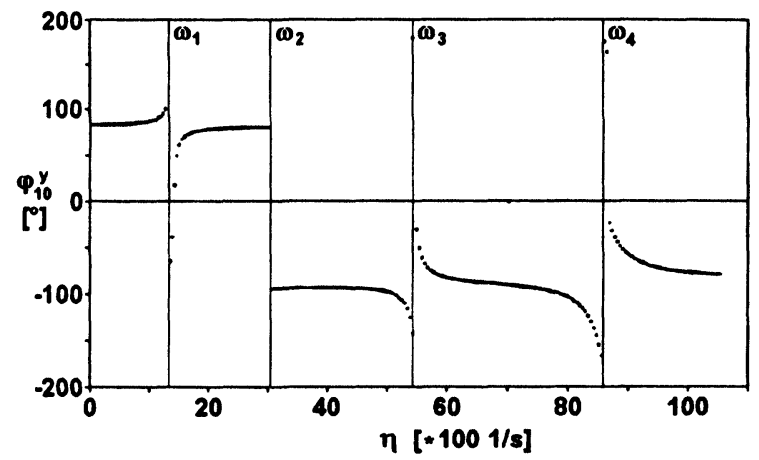

FIGURE 4 Amplitude curve and phase shift curve of constant undamped system against turbine frequency. 

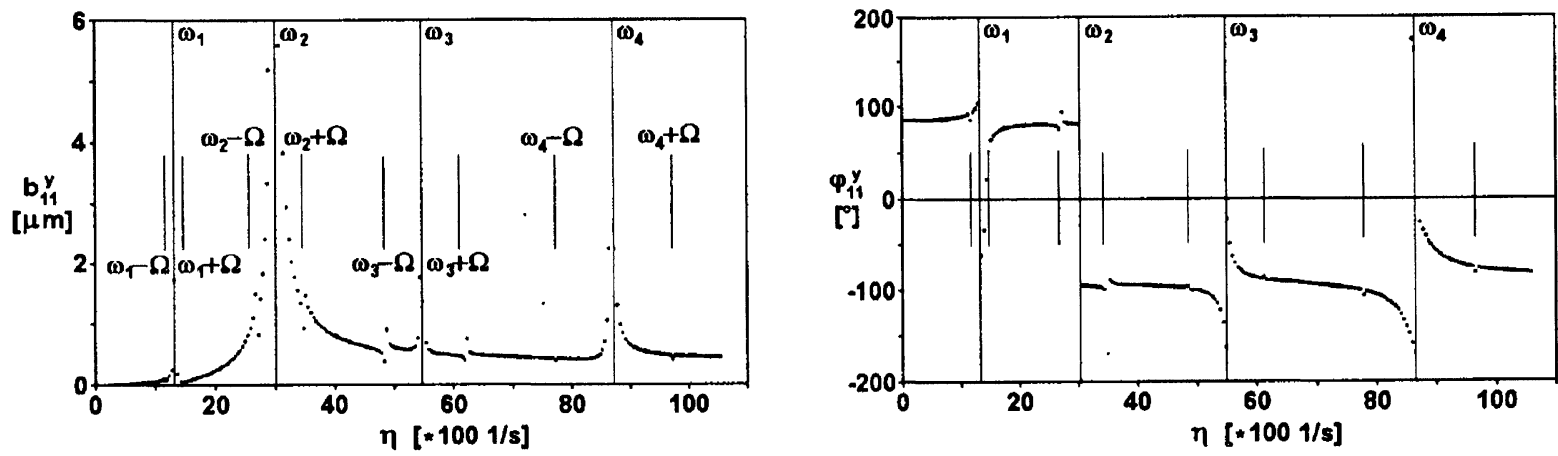

FIGURE 5 Amplitude curve and phase shift curve of undamped system against turbine frequency (first order approximation).
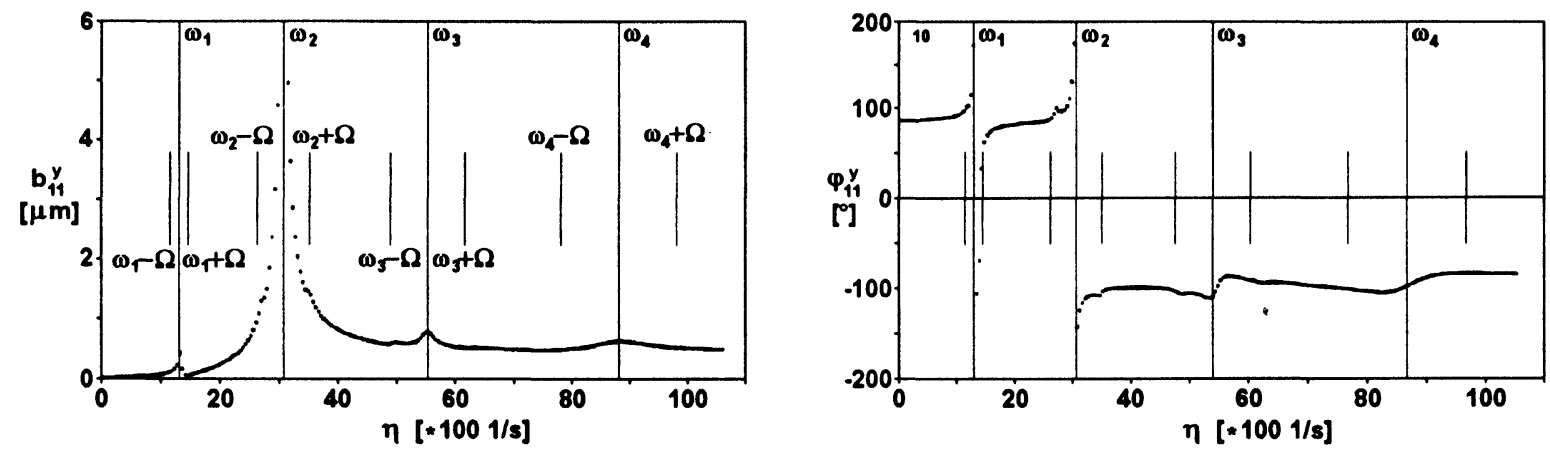

FIGURE 6 Amplitude curve and phase shift curve of damped system against turbine frequency (first order approximation).

solutions) are shown in Figure 4 against spinning turbine frequency $\eta$. Only four isolated forced resonances in the case of homogeneous elasticity of the twin disc coating are shown. These two plots for the undamped system without parameter excitation are used as references. In Figure 5 two neighbouring interaction resonances can be detected beside each main forced resonance in the undamped case, while in the damped case, Figure 6, only little information about these interactions are recognizable. In the last four plots hull curve representations of the centre part of the amplitude vector

$$
\overline{\mathbf{b}}_{s s}=\sum_{q=-1}^{1} \sum_{p=-P}^{P} \overline{\mathbf{b}}_{q p}
$$

in $Y$-direction are given for different approximation orders $P$ in the undamped case, see Figures 7, 9, and the damped case, Figures 8,10 , respectively. The figures demonstrate the dominant appearance of the main parametric resonance $\omega_{2}=2 \Omega$ (compare with Figure 3 ) for $\eta \approx 10000[1 / s]$. It should be known that in Figures 7-10 the phase shift of damping and stiffness inhomogeneities of the twin-disc has been changed from $180^{\circ}$ to $0^{\circ}$ by changing the parameter intensity coefficients of the second twin-disc pair to $\alpha_{t 2}^{c k}=+0.3 /+0.1 /+0.05, \beta_{t 2}^{c k}=+0.3 /+0.1 /+$

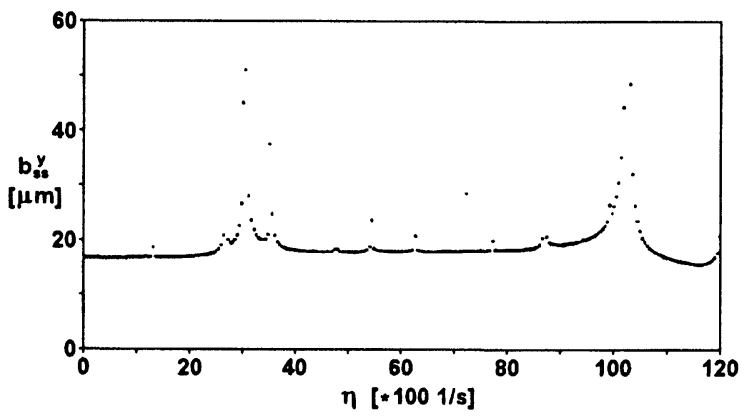

FIGURE 7 Hull curve of undamped system against turbine frequency (first order approximation). 


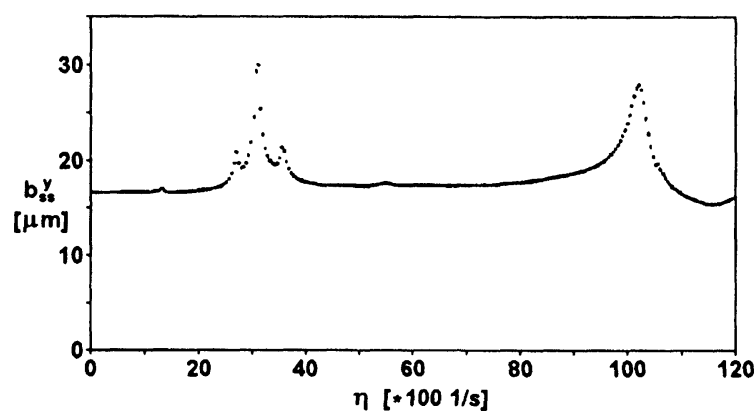

FIGURE 8 Hull curve of damped system against turbine frequency (first order approximation).

0.05. Parallel to the afore-mentioned effects further resonances appear and can be characterized with the help of a completed Figure 3.

Stability information Complementary to this an approximate stability analysis, for example discussed in Neumann et al. [1994], separates stable from unstable parameter regions. Here unstable solutions are situated in parameter regions with high amplitudes, see Figure 3, or it follows from high damping that the most unstable regions dissapear.

\section{CONCLUSIONS}

In this paper the results of the method of enlarged systems for the calculation of periodic parameter excited problems including the interaction with forced excitations have been presented. The central idea and

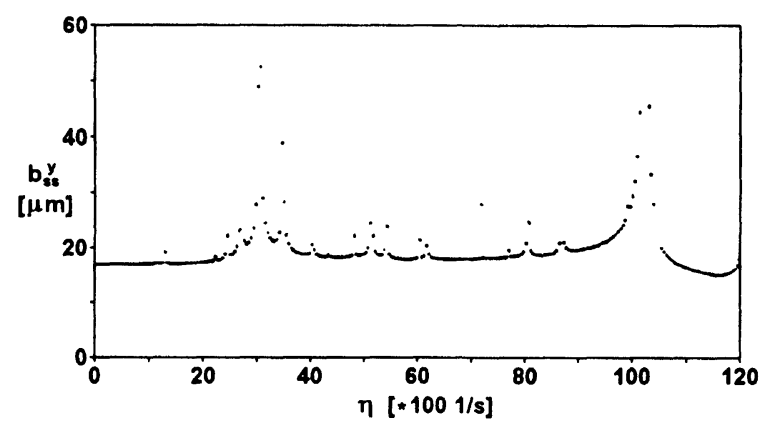

FIGURE 9 Hull curve of undamped system against turbine frequency (10th order approximation).

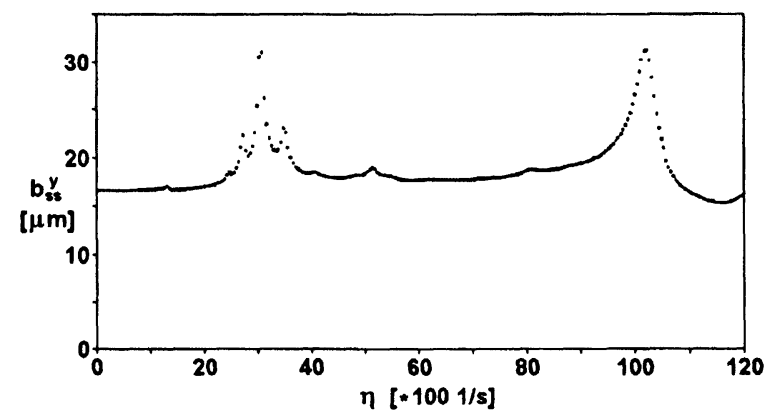

FIGURE 10 Hull curve of damped system against turbine frequency (10th order approximation).

the structure of the enlarged system have been analysed. In general the enlargement of the system equations allows:

- the approximate calculation of the characteristic exponents, including a stability definition and a new interpretation of the well known conditions of parameter and combination resonances,

- the approximate calculation of the complete homogeneous solution,

- the approximate calculation of the inhomogeneous solution and

- a complete overview of resonance phenomena resulting from interaction of parametric and forced excitation with in general non commensurable basic frequencies.

Exceptionally the method is based only on the procedures of constant systems the engineer is familiar with.

General homogeneous and inhomogeneous solutions have been derived and applied to a realization of the high speed twin-disc rotor-bearing-system of a textile spinning turbine. The new effects of interaction of forced and parametric excitation on the rotor vibration have been discussed.

\section{Acknowledgment}

This investigation was supported by the W. Schlafhorst AG \& Co., Mönchengladbach. The author thanks Dr.-Ing. H. Socha, head of Dept. of ST I, for his unbureaucratic involvement. 


\section{NOMENCLATURE}

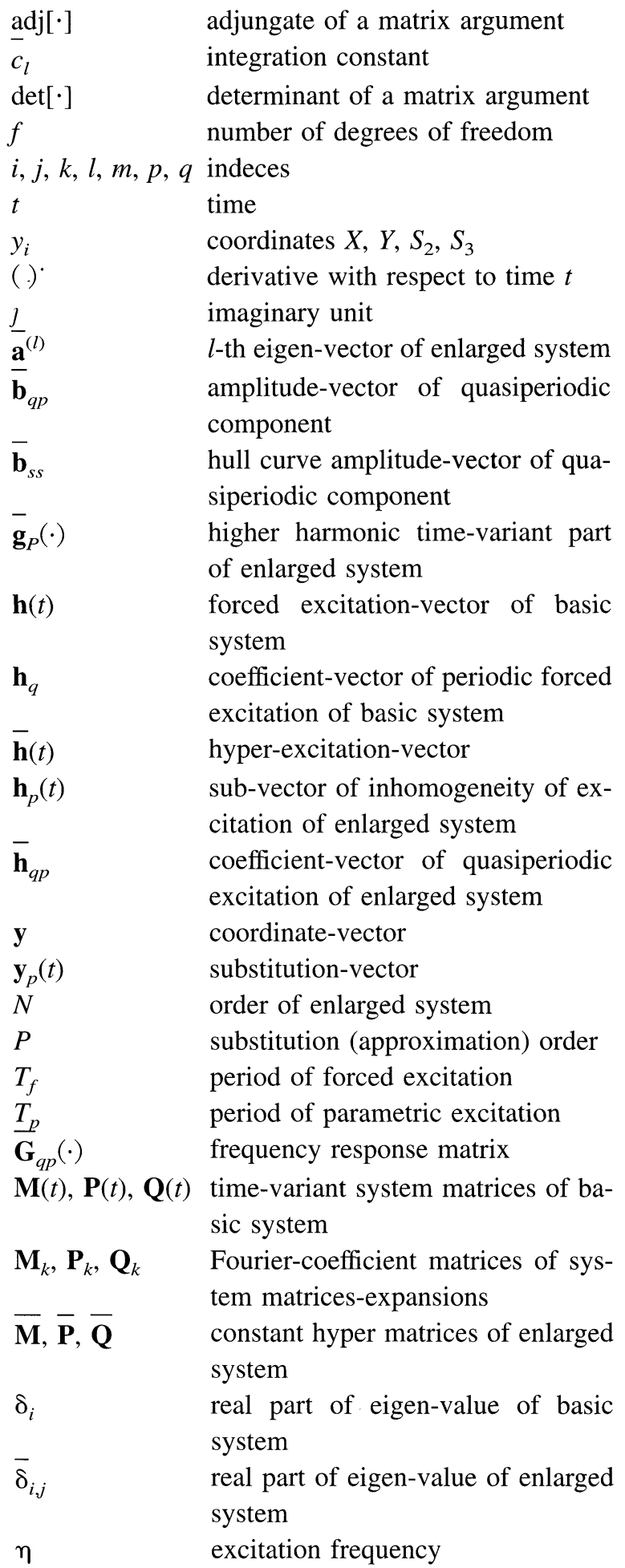

$\begin{array}{ll}\bar{\lambda}_{l} & \text { eigen-value of enlarged system } \\ \omega_{i} & \text { imaginary part of eigen-value of ba- } \\ \bar{\omega}_{i, j} & \text { sic system } \\ \bar{\Delta}_{i, j} & \text { imaginary part of eigen-value of en- } \\ \Omega & \text { larged system } \\ & \text { eigen-value approximation error } \\ & \text { parametric frequency }\end{array}$

For additional information see also Table II.

\section{References}

Bolotin, V.V., 1964. The Dynamic Stability of Elastic Systems, Holden-Day, San Francisco

Diekhans, G., 1981. Numerische Simulation von parametererregten Getriebeschwingungen, Diss. TH Aachen

Friedemann, C.E., Hammond, C.E., Woo, T.H., 1977. Efficient Numerical Treatment of Periodic Systems with Application to Stability Problems, Int. Journal for Numerical Methods in Engineering, Vol. 11, pp. 1117-1136

Hanzel, J., 1985. Mathematisches Modell der Stützrollenlagerung des Spinnrotors der Rotor-Spinnmaschine mit Drehzahlen bis 120000 min $^{-1}$, Diss. Usti n. Orl

Laschet, A., 1988. Simulation von Antriebssystemen, Fachberichte Simulation, Bd.9, Berlin-Heidelberg-New York-London-ParisTokyo, Springer-Verlag

Müller, P.C., Schiehlen, W.O., 1976. Linear Vibrations, Akademische Verlagsgesellschaft, Wiesbaden

Naab, K., Weyh, B., 1988. Zur Bestimmung der charakteristischen Exponenten parametererregter Systeme, Zeitschrift für Angewandte Mathematik und Mechanik, Vol. 68, pp. T92-T94

Neumann, U., Weyh, B., 1994. Charakteristische Exponenten und homogene Lösung periodisch parametererregter Systeme, Zeitschrift für Angewandte Mathematik und Mechanik, Vol. 74, pp. T48-T51

Socha, H., Wintzen, K., 1991. Development Report, W. Schlafhorst $\mathrm{AG} \& \mathrm{Co}$

Vieweger, R., Weyh, B., 1993. Erzwungene quasiperiodische Schwingungen linearer parametererregter Systeme, Zeitschrift für Angewandte Mathematik und Mechanik, Vol. 74, pp. T83-T85

Weck, M., Hartel, R., 1984. Analyse des dynamischen Verhaltens von Rotorspinnturbinen nach dem Signatur- und Modalanalyseverfahren, VDI-Berichte 536, pp. 205-219

Weyh, B., 1989. Calculation of Stability Maps of Time-Varying Systems, Mechanical Vibration and Noise, (Diagnostics, Vehicle Dynamics and Special Topics) 1989, Proc. 12th Biennial ASME Conf. Montreal/Canada, pp. 293-298

Weyh, B., 1990. Rapid Stability Analysis of Parameter-Excited Large Systems, in Dynamics of Rotating Machinery, Part II, Eds. Kim, J. H., Yang, W.-J., pp. 303-314, Hemisphere Publishing Corporation, New York-Washington-Philadelphia-London

Weyh, B., Kostyra, H., 1991. Direct Floquet-Method for Stability Limits Determination, I-Theory, II-Application and Phenomena, Mechanism and Machine Theory, Vol. 26, No. 2, pp. 123-144

Weyh, B., 1993. Vibration analysis of open-end spinning rotors, Proc. Asia-Pacific Vibration Conf., Kitakyushu/Japan, Vol. 2, pp. 388-393

Yakubovich, V.A., Starzhinskii, V.M., 1975. Linear differential equations with periodic coefficients, John Wiley \& Sons, New York-Toronto 


\section{ait \\ ENERGY MATERIALS}

M A N E Y publishing

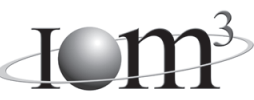

\section{Materials Science \& Engineering for Energy Systems}

Maney Publishing on behalf of the Institute of Materials, Minerals and Mining

The Institute of Materials, Minerals \& Mining

Economic and environmental factors are creating ever greater pressures for the efficient generation, transmission and use of energy. Materials developments are crucial to progress in all these areas: to innovation in design; to extending lifetime and maintenance intervals; and to successful operation in more demanding environments. Drawing together the broad community with interests in these areas, Energy Materials addresses materials needs in future energy generation, transmission, utilisation, conservation and storage. The journal covers thermal generation and gas turbines; renewable power (wind, wave, tidal, hydro, solar and geothermal); fuel cells (low and high temperature); materials issues relevant to biomass and biotechnology; nuclear power generation (fission and fusion); hydrogen generation and storage in the context of the 'hydrogen economy'; and the transmission and storage of the energy produced.

As well as publishing high-quality peer-reviewed research, Energy Materials promotes discussion of issues common to all sectors, through commissioned reviews and commentaries. The journal includes coverage of energy economics and policy, and broader social issues, since the political and legislative context influence research and investment decisions.

\section{CALL FOR PAPERS}

Contributions to the journal should be submitted online at http://ema.edmgr.com

To view the Notes for Contributors please visit: www.maney.co.uk/journals/notes/ema

Upon publication in 2006, this journal will be available via the Ingenta Connect journals service. To view free sample content online visit: www.ingentaconnect.com/content/maney

For further information please contact:

Maney Publishing UK

Tel: +44 (0)113 2497481 Fax: +44 (0)1132486983 Email: subscriptions@maney.co.uk

or

Maney Publishing North America

Tel (toll free): 8662975154 Fax: 6173546875 Email: maney@maneyusa.com

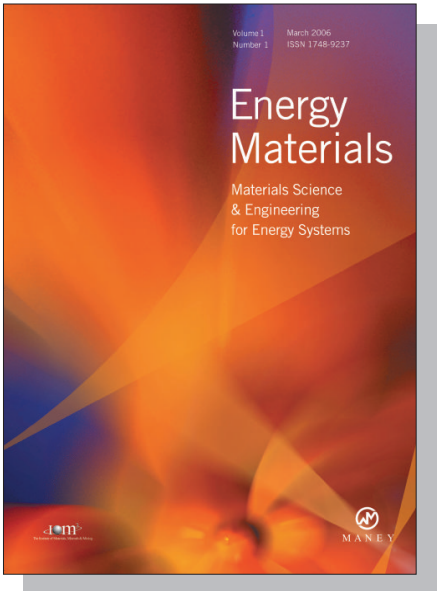

EDITORS

Dr Fujio Abe

NIMS, Japan

Dr John Hald, IPL-MPT, Technical University of Denmark, Denmark

Dr R Viswanathan, EPRI, USA

\section{SUBSCRIPTION INFORMATION}

Volume 1 (2006), 4 issues per year

Print ISSN: 1748-9237 Online ISSN: 1748-9245

Individual rate: $£ 76.00 / U S \$ 141.00$

Institutional rate: $£ 235.00 /$ US $\$ 435.00$

Online-only institutional rate: $£ 199.00 / U S \$ 367.00$

For special $\mathrm{IOM}^{3}$ member rates please email

subscriptions@maney.co.uk

\section{For further information or to subscribe online please visit www.maney.co.uk}



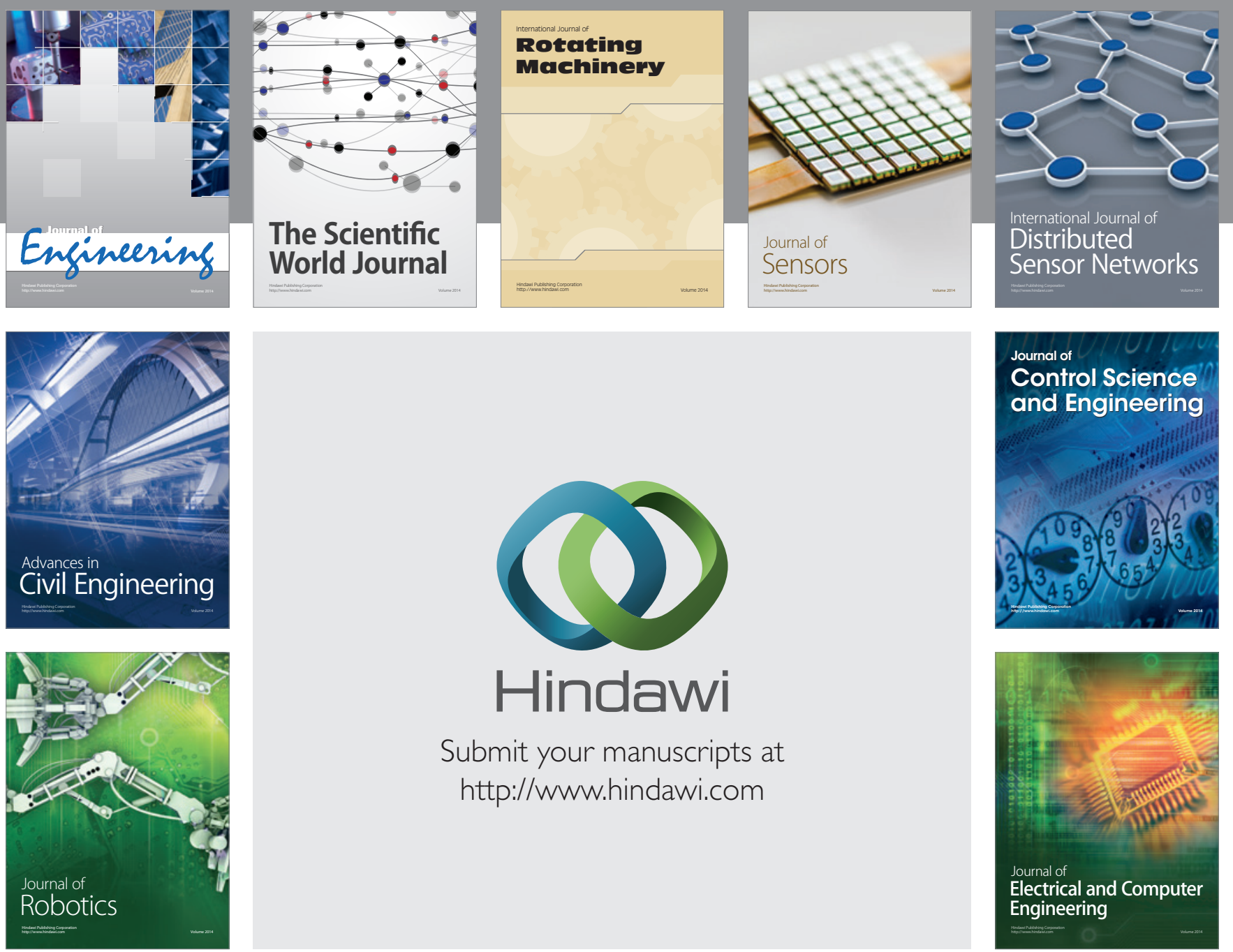

Submit your manuscripts at

http://www.hindawi.com
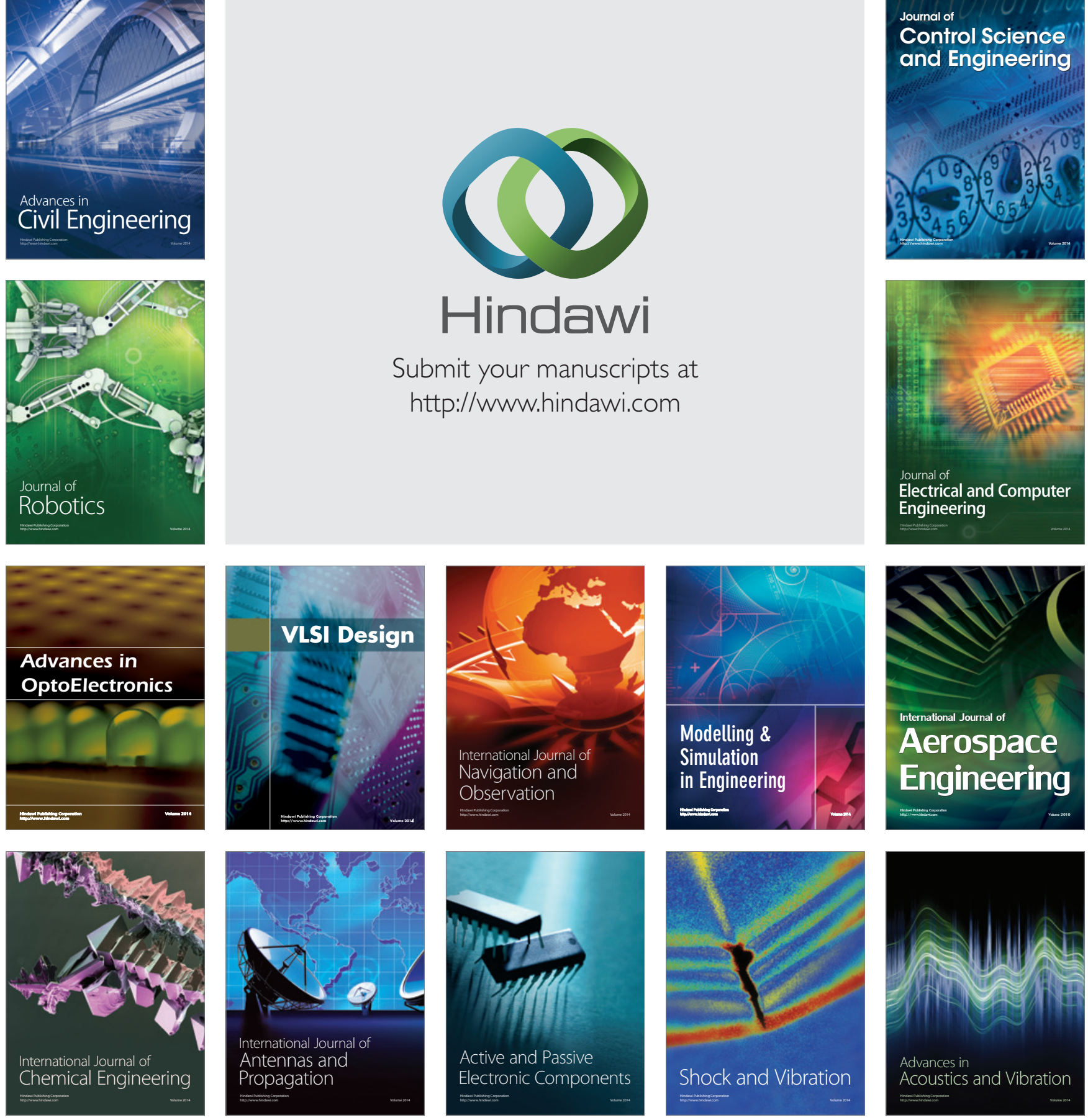